\title{
Evaluation METHOdology OF MiMO Networks Performance over Rayleigh FADING
}

\author{
Eleftherios Stergiou, Constantinos Angelis and Spiridoula V. Margariti \\ Department of Informatics and Telecommunications, University of Ioannina, \\ GR-47100 Arta, Greece
}

\begin{abstract}
Today, the explosive growth of Multiple Input Multiple Output (MIMO) systems has resulted in a high data rate and consequently permits the operation of a variety of applications. The MIMO networks are multiparameter systems, so the choice of a suitable MIMO network in wireless communications is a complex issue. In this paper, a multi-factoring evaluation and comparison framework was introduced and applied to MIMO systems. The proposed methodology is based on a general distance function, named the General Evaluation Factor. This method was applied to MIMO networks that operate over Rayleigh fading channels with different antenna nodes and spacing. The implementation of this method was based on different capacities and cost values. Nevertheless, while only two factors (capacity and cost) were studied in this paper, the proposed approach was able to incorporate additional performance metrics that might be essential for many wireless system designs. The presented framework and results aspire to be useful for network engineering, especially when finding a balance between contradictory factors (e.g. cost and performance metrics) on MIMO networks.
\end{abstract}

\section{KEYWORDS}

MIMO systems, Capacity of channel, Cost analysis, Optimization, Rayleigh fading channel, Evaluation

\section{INTRODUCTION}

Over the last decade, technological advances in wireless communication have been particularly rapid. An excellent overall spectral activity of a wireless communication system may be accomplished at several manners of system design[1-2], for example, by minimizing the diameter of cell area and the co-channel reuse cell's diameter or at network level decreasing the cell area or by using advanced channel allocation schemes that increase the overall amount of transferred data. A key feature characterizing all modern wireless systems that are gradually being implemented is their increased complexity. The MIMO technology is widely considered as one key enabler for filling the capacity gap towards the next generation of wireless communications. The choice of MIMO systems in mobile networks depends on a large number of factors. Consequently, the decision to choose the optimal MIMO system is an open and complex problem. This paper will attempt to answer this multi-factorial question.

\subsection{MIMO systems}

In the digital communication area, Multiple-Input Multiple-Output (MIMO) systems are one of the most significant technical milestones in wireless communications. MIMO systems are defined as complex systems that contain multiple transmit antennas and multiple receive antennas. In general, many studies show that MIMO systems have the ability to provide a very high capacity 
which, in most cases rises in an approximately linear fashion with the number of antennas $[3,4]$. Until now, various types of MIMO's architectures have also been implemented by relevant industrial companies for the wireless communications market. An example of a MIMO network is shown in Fig.1.

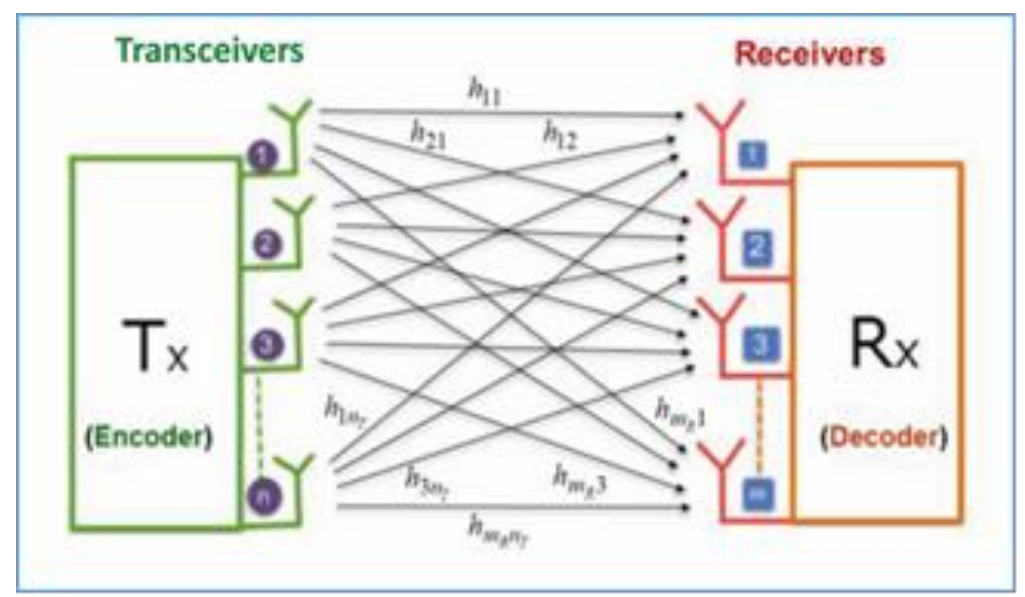

Figure 1. Typical view of a MIMO network

\subsection{Previous work}

As MIMO systems have been actively involved in the structure of wireless networks over the past 10 years, many related studies have been published and mainly focusing on performance metrics. For example, [3-10] study performance calculation approaches of various MIMO systems under different conditions and provide corresponding results. In order to adequately cover the area between source and destination, a number of antennas are required. The number of antennas on each side can vary. Today the most usual number of antennas for MIMO systems is 4, 8, or 16 . Nevertheless, the requirements of current 5 th generation networks are extremely demanding, so the number of antennas that are used has grown. s. Massive MIMO is the term used for MIMO systems that use more than 64 antennas and such systems are required by the 5 th generation of wireless networks [11-15]. Today the usual number of antennas for massive MIMO is 64, 128 or 256. This study will be limited to the study of simple systems, but this approach can be applied without any special changes, including in massive MIMO systems. Many studies investigate other performance factors of MIMO systems such as the bit error rate [14], reliability [16], or design issues [17] and so on.

The cost of a MIMO system increases exponentially according to its dilation, and is in contrast to its performance. A good example of MIMO cost-effectiveness is presented by Weiqiang Tan et al. [18]. However, the authors limit their study to a hybrid structure of multi-user massive MIMO. The wireless system consist of digital and analog domain, where the first uses the zero-forcing (ZF) precoding concept while the second utilizes Discrete Fourier Transform (DFT) method, which considerably minimizes the cost of hardware and the consumption of power. In addition, MIMO cost approaches were studied by Caire et al. [19] and Liu et al. [20]. But both of those studies are rather technical comparisons and not real economic approaches. A real econometric study was published by Bouras et al. [21] and elements from this study were used as values in the current approach. 


\subsection{The scientific gap and the contribution of the current work}

MIMO technology is really a multi-parameter problem. A MIMO system depends on several different parameters that are often opposed to each other. Various factors can be considered as typical parameters in a MIMO system, for example, performance metrics (e.g. capacity, bandwidth, BER), energy consumption, economic costs, and so on. As more parameters are involved in a MIMO system, the optimal choice of system becomes more complex. In the literature there are various works that study different problems separately, usually investigating them thoroughly and yielding results manner for one or two system parameters. Hence, the final question remains, what is ultimately the best choice of a system among many?

The criteria that can be used in any study case depend on the ultimate purpose of engineers and the weight of criteria desired each time. Our approach proposes a general method that can be applied to various MIMO technologies, and other similar technologies that have a similar structure. This flexible method can be implemented for different technical and financial scenarios. Nevertheless, here the method is exemplified on MIMO systems with Rayleigh fading channels and their corresponding cost, and the proposed method does not lose its generality. In other words, our work attempts to bridge the gap that arises from the contradictory metrics in the networking area MIMO, making their overall evaluation extremely complex.

The rest of this paper is organized as follows: in Section 2 we present the Rayleigh fading channel and give details of the calculation channel's capacity with a corresponding case. In Section 3, a cost analysis of MIMO systems is presented, depending on the system's complexity and market data. In Section 4, the methodology for MIMO systems evaluation is presented. This approach takes into consideration two contradictory factors (capacity; cost) of MIMO systems. In Section 5 , numerical results are illustrated that are involved with MIMO systems operating over a Rayleigh fading channel, while in Section 6 conclusions are drawn and future work is outlined.

\section{Mimo Systems, Rayleigh Fading Channel and Channel's CAPACITY}

\subsection{MIMO fundamentals issues}

Contrary to ordinary communication systems which use one transmit and one receive antenna, MIMO systems employ multiple antennas at both ends of communication channel (see Figure 1). Therefore, the MIMO channel has to be described for all pairs of transmit and receive antennas. Generally speaking, let us consider an $n_{T} \times n_{R}$ MIMO system, where $n_{T}$ and $n_{R}$ are the number of transmit and receive antennas, respectively. From a system level point of view, a linear timevariant MIMO channel can be described by an $n_{T} \times n_{R}$ channel matrix, as follows:

$$
H(t, \tau)=\left(\begin{array}{cccc}
h_{11}(t, \tau) & h_{12}(t, \tau) & \cdots & h_{1 n_{R}}(t, \tau) \\
h_{21}(t, \tau) & h_{22}(t, \tau) & \cdots & h_{2 n_{R}}(t, \tau) \\
\vdots & \vdots & \vdots & \vdots \\
h_{n_{T} 1}(t, \tau) & h_{n_{T} 2}(t, \tau) & \cdots & h_{n_{T} n_{R}}(t, \tau)
\end{array}\right)
$$

where $h_{i j}(t, \tau)$ depicts the time-variant impulse response between the $j^{\text {th }}$ transmit antenna $\left(j=1, \ldots, n_{T}\right)$ and the $i^{t h}$ receive antenna $\left(i=1, \ldots ., n_{R}\right)$. Spatially, there is no discrimination between separate antennas and different polarization modes of the same antenna. 
In cases where polarization-diverse antennas are studied, each element of the matrix $\mathrm{H}(t, \tau)$ has to be substituted by a polarimetric submatrix, which has the effect of rising the overall number of antennas that are included in the system. The factors of the channel's matrix (1) comprise the effects of the antennas (e.g. type, configuration) and frequency filtering depending on the bandwidth.

According to MIMO theory [1, 22-23], the aforementioned can be used to form an overall MIMO input-output relation between the $n_{T}$-length transmit signal vector $x(t)$ and the $n_{R}$-length receive signal vector $y(t)$ as follows:

$$
y(t)=\int_{\tau} H(t, \tau) \cdot x(t-\tau) d \tau+n(t)
$$

where $n(t)$ models the noise and interference.

Assuming that the channel is time-invariant, then the dependence of the channel matrix on $t$ vanishes (so it can be written: $H(\tau)=\mathrm{H}(t, \tau)$ ). Moreover, assuming the channel is frequency flat, there exists only one single flow, which can be described by $H$. Following all the above, formula (2) can be written more simply as:

$$
y(t)=H \cdot x(t)+n(t)
$$

The elements $h_{i, j}$ of the channel matrix $H$ can be considered as deterministic or random. In this study we will investigate wireless networking, which involves the Rayleigh distribution of channel matrix elements. This is because in most situations the Rayleigh distribution is the most representative case for non-line of sight radio propagation.

\subsection{Rayleigh fading channel}

Rayleigh fading is a representative example when in the coverage area of an antenna there are several objects that cause scattering of the radio signal before it reaches the receiver's side.

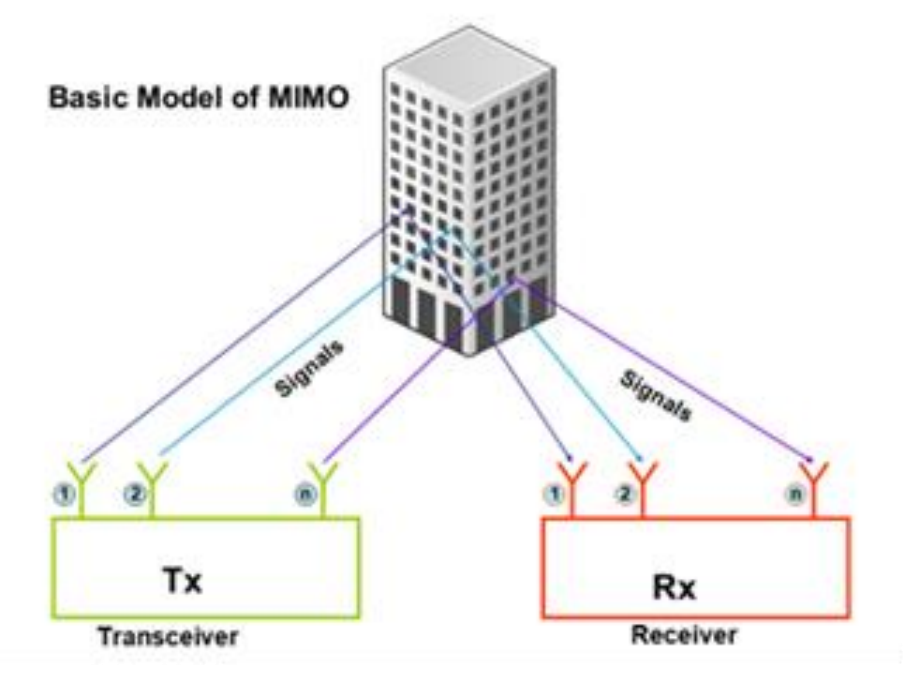

Figure 2. A MIMO network example with Rayleigh fading channel 
In wireless transmission, the envelope of the carrier signal accepted at destination side is Rayleigh distributed. This fading may be due to multipath with or without the Doppler Effect. A Rayleigh fading model considers that the magnitude of a signal that has been transferred through a transmission channel or medium will vary frequently and randomly, or fade, according to a Rayleigh distribution. Usually, the Rayleigh fading is applied in circumstances when there is less or no dominant propagation along a visual line from the transmitter to the receiver. The MIMO system is a typical communication technology whose radio signals are scattered in the environment - because of objects in the environment - and therefore the Rayleigh fading is a suitable model for this. The signal's behavior of this case is described by the central limit theorem. This theorem maintains that, if there is enough scattering, then the channel's impulse response can be represented satisfactorily by a Gaussian model, without affecting the distribution of the distinct entities of the system.

If the resulting scatter does not have a dominant component, then such a process will present a zero mean and phase evenly distributed between 0 and $2 \pi$ radians. Consequently, the envelope of the channel response can be considered as Rayleigh distributed [27]. Rayleigh distribution represents a continuous probability distribution for nonnegative random variables. Frequently, the representation of the channel's distortion parameters: gain and phase elements are based on complex numbers. The Rayleigh fading is determined accepting that the real and imaginary components of the response are represented by identically and independent distributed zero-mean Gaussian model, so that the overall of the response is an aggregation of two such parts.

\subsection{Channel's capacity}

In general, the MIMO system's capacity $C\{$ bits $/ s\}$ is denoted by the upmost possible transmission rate when the error probability is in lowest level of values. Initial investigations relating to the ability of the MIMO ergodic capacity did not take into account any limitations on entry signals. Based on this assumption, Foschini and Gans [23] and Telatar [23] separately obtained a formula for the ergodic capacity. Moreover, Vucetic et al. [1] present the capacity of MIMO networks operating in fast and block Rayleigh fading channels, as follows:

$$
C=E\left\{W \cdot \log _{2}\left[\operatorname{det}\left(I_{r}+\frac{P}{n_{T} \cdot \sigma^{2}} Q\right)\right]\right\}
$$

where $C$ depicts the channel capacity (bits/s), $W$ is the bandwidth of the channel $(\mathrm{Hz}), r$ is the rank of the channel's matrix $H, P$ depicts the average total transmitter power, $n_{T}$ is the number of transmitted antennas, $\sigma^{2}$ is the identical noise on each receive link and the matrix $\mathrm{Q}$ is the Wishart matrix defined as follows:

$$
Q=\left\{\begin{array}{ll}
H H^{H} & \text { for } n_{R}<n_{T} \\
H^{H} H & \text { for } n_{R} \geq n_{T}
\end{array} .\right.
$$

In the case of a flat Rayleigh fading channel, the coefficients of channel are random while in Gaussian channel the coefficients are considered fixed. Here zero-mean coefficients of the channel are assumed. That means complex Gaussian random variables with variances of $1 / 2$ per dimension (real and imaginary). So, each channel coefficient has a Rayleigh distributed magnitude and uniformly distributed phase. In addition, the expected value of the squared magnitude is equal to one: hence: $E\left[\left|h_{i, j}\right|^{2}\right]=1$, where $h_{i, j}$ depicts the channel's coefficients 
between the $j^{\text {th }} T_{x}$ antenna $\left(j=1, \ldots ., n_{T}\right)$ and the $i^{\text {th }} R_{x}$ antenna $\left(i=1, \ldots ., n_{R}\right)$. In the next part of this study, the coefficients of a channel are assumed to be known at the receiver, but unknown at the transmitter. That means that the transmitted power per $T_{x}$ antenna is assumed to be identical and equal to $P_{t j}=\frac{P}{n_{T}}$ for $j=1, \cdots, n_{T}$, where $P$ depicts the average total transmitter power.

Then the following scenarios are considered. These cases have been widely considered in the scientific literature [1,23].

- The matrix $\mathbf{H}$ of channel coefficients is random and its entries change randomly during every symbol time slot. This case is referred to as a fast flat Rayleigh fading channel.

- Matrix $\mathbf{H}$ is random and its items become different at random after a fixed period, which consist of a fixed number of time slots. This case is referred to as the block flat Rayleigh fading channel.

\subsection{Capacity of MIMO wireless systems operating in fast and block Rayleigh fading channels}

The above Eq. (4) can be estimated quite well with the help of Laguerre polynomials. This approach is presented by Vucetic et al. [1] (see section 1.6.1) and by Telatar [23] (see Theorem 2). The capacity factor can be written as:

$$
C=W \int_{0}^{\infty} \log _{2}\left(1+\frac{P}{n_{T} \sigma^{2}} \lambda\right)_{k=0}^{m-1} \frac{k !}{(k+n-m) !} \times\left[L_{k}^{n-m}(\lambda)\right]^{2} \cdot \lambda^{n-m} \cdot e^{-l} d \lambda
$$

where $n=\max \left(n_{T}, n_{R}\right), m=\max \left(n_{T}, n_{R}\right)$, and

$$
L_{k}^{n-m}(\lambda)=\frac{1}{k !} e^{\lambda} \lambda^{m-n} \frac{d^{k}}{d \lambda^{k}}\left(e^{-\lambda} \lambda^{n-m+k}\right)
$$

is the Laguerre polynomial of order $k$.

Furthermore, by increasing $m$ and $n$, but keeping the ratio $\tau=\frac{n}{m}=$ const, we have the following limit:

$$
\lim _{n \rightarrow \infty} \frac{C}{m}=\frac{W}{2 \pi} \int_{v_{1}}^{v_{2}} \log _{2}\left(1+\frac{P \cdot m}{n_{T} \sigma^{2}} v\right) \sqrt{\left(\frac{v_{2}}{v-1}\right) \cdot\left(1-\frac{v_{1}}{v}\right)}
$$

Where $v_{1}=(\sqrt{\tau}-1)^{2}$ and $v_{2}=(\sqrt{\tau}+1)^{2}$.

\section{Example scenario}

Then let us take as an example of the MIMO system the following case: $n_{T}=1$ and $n_{R} \geq 2$. Hence Eq. (6) can be rewritten:

$$
C=W \frac{1}{\left(n_{R}-1\right) !} \int_{0}^{\infty} \log _{2}\left(1+\frac{P}{\sigma^{2}} \lambda\right) \lambda^{n_{R}-1} e^{-\lambda} d \lambda
$$


When the ${ }^{n_{R}}$ is heightened, then the capacity tends in an asymptotic value:

$$
\lim _{n \rightarrow \infty} C=W \cdot \log _{2}\left(1+\frac{P_{n_{R}}}{\sigma^{2}}\right)
$$

This reveals that, when the number of antennas is large, the capacity of the receive diversity system in fast or block Rayleigh fading channels tends to the capacity of the receive diversity system in additive white Gaussian noise (AWGN) channels.

\section{COST APPROACH OF MIMO SySTEMS}

According to Bouras et al.'s [21] analysis, the overall Cost of using a MIMO system can be distinguished in two main types:

- FCOST (Fixed Cost): the cost of acquiring and installing a MIMO system, which is considered as a stable cost, and

- VCOST (Variable Cost): the cost of the daily operation and maintenance. In a running system, the cost of day-to-day management, power consumption, possible repayments of capital, bank interest, other bank charges, etc. can be added.

The two categories of expenditure are extremely different and because the individual cost components are constantly changing, it is quite difficult to calculate the real cost of each class of expense. In the current work, it is carried out a study about that the fixed costs are paid once at system start-up, while variable costs are considered as expenses paid annually and the amount of the expenditure then depends on a number of other external factors.

Let us suppose, in general, that we have a system with a multi base-station MIMO system with $B$ number of base-stations (BS). Also, let $C_{B S}$ depict the cost of a BS and $C_{E P C}$ the cost of the Evolved Packet Core (EPC). Then, the Total Cost of this multi base-station system can be written as:

$$
B \cdot\left(C_{B S}+C_{E P C}\right)
$$

In addition, the MIMO technology uses a number of antennas at the source and destination sides. This number of antennas should be considered as well. Let us consider as $n_{T}$ and $n_{R}$ the antennas' number at the source and destination sides respectively. Hence, if the cost of an antenna is equal to $A$ cost units, then the Total Cost of antennas will be:

$$
A \cdot\left(n_{T}+n_{R}\right)
$$

Subsequently, the overall fixed Cost of a MIMO system is:

$$
\mathrm{FCOST}=B \cdot\left(C_{B S}+C_{E P C}\right) \cdot A \cdot\left(n_{T}+n_{R}\right)
$$

For simplicity in this study, the variable Cost of the MIMO system (VCOST) is ignored. Then, based on market data sampling, the following typical values are shown in Table 1 . The values of Table 1 are also confirmed in Table 2 in the study by Buras et al. [21], who present cost parameters and system variables related to MIMO systems. The continuation of the current cost study will be based on Table 1 . 
Table 1. Measured performances in different operating conditions.

\begin{tabular}{|l|l|c|}
\hline \hline \multicolumn{2}{|l|}{ Typical Purchase cost of MIMO components and parameters } \\
\hline \hline Parameter & Description & Value range for SA \\
\hline General costs & {$[500,1500]$ Euros } \\
\hline$C_{B C}$ & Capital cost for single BS & {$[55,165]$ Euros } \\
\hline$C_{E P C}$ & $\begin{array}{l}\text { Core network's capital cost for deployment of } \\
\text { a single BS }\end{array}$ & {$[1,100]$ Euros } \\
\hline$B / N$ & The total number of BSs and EPCs needed & $\begin{array}{l}{[2,4,8,16,64,128,256,} \\
512]\end{array}$ \\
\hline \multicolumn{2}{|l|}{ Common quantized usage values of MIMO system antennas } \\
\hline$n_{T}$ & $\begin{array}{l}\text { Factor associated to the MIMO antennas at the } \\
\text { transmit side }\end{array}$ \\
\hline
\end{tabular}

The cost of BS stations is slowly changing in relation to antenna costs, and is changing at an ever faster pace each year, and sometimes even more often. This is because the research and development of BS is a more complex process, highly specialized and costly. This leads us in the current analysis to obtain as values of the BS the values from Table 1 and to consider the cost value of a MIMO antenna is equal to A (Euros). Hence, the final cost units are expressed as a function of cost A (Euros). We should not forget that when the cost values are normalized then the resulting values, due to the simplification, will be perfectly correct and independent of the antenna's cost A.

Taking into account formula (13) and the values of Table 1, the cost for the three different scenarios (MIMO architectures, explained in Section 5) are calculated.

\section{Evaluation of Multi Magnitudes DePendent on MIMO Systems}

The choice of MIMO systems in wireless networks depends on a number of separate factors. In the current section, a general methodology of multi-factoring system selection is proposed. The method considers a set of parameters (e.g. performance measures, complexity, or cost factors); these parameters can be portrayed onto a multi-dimensional space representing the factors to be evaluated. Consequently, this will allow us to compare MIMO systems with different complexities and performance magnitudes. To clarify, this method is not limited to two metrics as is presented here - but is more general and can include additional factors. Nevertheless, these two metrics are selected because they are widely used in the literature. Generally, evaluating a communication system, be it hardware or software, requires a full study of the costs. The basic demand in terms of cost remains the need to be as small as possible. On the other hand, the performance of a system (e.g. capacity) is a magnitude that we want to be as large as possible. There are many relative studies on MIMO systems in the literature [4-10].

\subsection{General Evaluation Factor (GEF)}

Next, we will explain how some factors related to the MIMO systems can be combined with a general performance factor. Generally, the evaluation factors can be divided into two major categories. The first category includes factors which are considered to have a positive effect when their values are maximized; the second comprises factors which are considered to result in a favourable action in the system when their values are minimized. The first category is named maximized factors, which is denoted as: $f^{\max }=\left\{f_{1}^{\max }, f_{2}^{\max }, \ldots \ldots ., f_{n}^{\max }\right\}$, while the other group is named minimised factors, $f^{\text {min }}=\left\{f_{1}^{\text {min }}, f_{2}^{\text {min }}, \ldots \ldots ., f_{n}^{\text {min }}\right\}$, where $\mathrm{n}$ is the number of MIMO system factors. 
For simplicity, let's limit the MIMO systems evaluation to minimized factors alone. In the case of minimized factor $f^{\text {min }}$, the general evaluation factor $(G E F)$ is calculated by the Euclidean distance of the performance projection value into a $n$ dimensions vector space, where dimensions represent various performance factors. In such a scenario, the $G E F$ can be formally portrayed as:

$$
G E F=\sqrt{\sum_{i=1}^{n}\left(f_{i}^{\text {min }}\right)^{2}}
$$

where $n$ is the maximum number of evaluated factors (minimised type).

Suppose we have two MIMO systems, MIMO system1 and MIMO system2, and their GEFs, $G E F 1$ and GEF2, respectively. In this case, if GEF1 < GEF2 then it can be said that MIMO system1 is better than MIMO system2. So, the above formula transforms the evaluation of a multimetrics evaluation problem in a unique function (metric). This function depicts the distance between the value of the $G E F$ and an ideal (non-realistic) MIMO system, for which the GEF value is equal to 0 . For make this more evident, let's consider an example of evaluating three MIMO systems, with two minimised type of metrics, $f$ and $f^{\prime}$. Figure 3 depicts in a twodimensional space the performance of the three MIMO systems: A, B, and C. Let $f_{A}$ and $f_{A}^{\prime}$ be the calculated values of factors for MIMO system A. Similarly, we have corresponding values of the same factors for MIMO systems B and C (See Table 2).

Table 2. Example of three MIMO system being evaluated with two metrics.

\begin{tabular}{|l|c|c|c|}
\hline \hline & System A & System B & System C \\
\hline metric $f$ & $f_{A}$ & $f_{B}$ & $f_{C}$ \\
\hline metric $f^{\prime}$ & $f_{A}^{\prime}$ & $f_{B}^{\prime}$ & $f_{C}^{\prime}$ \\
\hline$G E F$ & $G E F_{A}$ & $G E F_{B}$ & $G E F_{C}$ \\
\hline \hline
\end{tabular}

From Figure 3, it can be deduced that MIMO system A is more robust than MIMO systems B and C, as MIMO system A gives smaller $G E F$ values than systems B and C.

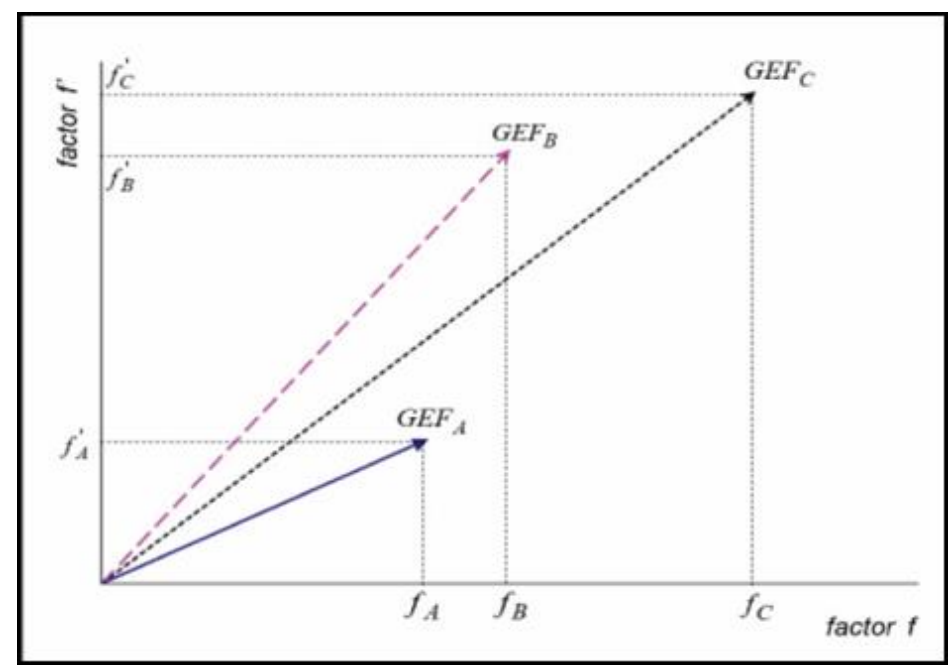

Figure 3. Example of three systems being evaluated with two metrics presented in a Euclidian space. 
$G E F_{A}$ is illustrated by the length the vector has in its coordinates $\left(f_{A} ; f_{A}^{\prime}\right)$ as are factors $G E F_{B}$ and $G E F_{C}$. Hence, in this case it is observed that the smaller the $G E F$ value, the better the corresponding MIMO system.

Frequently, however, the parameters have different types of measures and scaling. In order to integrate the evaluation, the value parameters must be normalised to a certain value. The resulting value may be the average value of the numbers, or the uppermost value for each factor.

The normalisation procedure using the maximum value provides the possibility of considering all factors with the same importance, and thus is capable of comparing different scaling metrics. If $N_{i}=\frac{f_{i}^{\min }}{\max \left(f_{i}^{\min }\right)}$, equation (14) can be replaced by the following equation:

$$
G E F=\sqrt{\sum_{i=1}^{n}\left(N_{i}\right)^{2}}
$$

Nevertheless, except the minimised factors all the factors other than the minimised factors should be considered simultaneously. As such, the system behaves better when factor $M_{i}=\frac{f_{i}^{\max }}{\max \left(f_{i}^{\max }\right)}$ tends to 1 . Subsequently, complementary factor $\left(1-M_{i}\right)$ tends to zero and can be included in formulae (15). So, formula (15), in order to include the maximised parameters, can be written as:

$$
G E F=\sqrt{\sum_{i=1}^{n}\left(N_{i}\right)^{2}+\sum_{j=1}^{m}\left(1-M_{i}\right)^{2}}
$$

where $n$ is the maximum number of evaluation factors (minimised type) and $m$ is the maximum number of evaluation factors (maximised type).

In our study, for example, the Capacity metrics can be considered as the maximised type of metric, while the Cost factor is considered as a minimising factor. Typically, in the systems that are affected by multi-parameters, when making decisions the importance of each metric is an initial design problem. Hence, the formulae of GEF can be written as:

$$
G E F=\sqrt{\sum_{i=1}^{n}\left(N_{i}\right)^{2} \cdot w_{i}+\sum_{j=1}^{m}\left(1-M_{i}\right)^{2} \cdot w_{j}},
$$

where $w_{i}$ is the weight of the minimised factor and $w_{j}$ is the corresponding weight of the maximised factor. This method can be applied in the same way in other scientific areas, such as Multistage Interconnection Networks (MIN) [24-25]. The studies of MINs are also a multifactorial issue. In this respect, the study of MIMO systems presents a similar problem.

The aforementioned factors (capacity and cost) will be used as examples for the validation of the proposed assessment methodology. 


\section{NUMERICAL RESULTS}

\subsection{Capacity in nuts of MIMO over Rayleigh fading channels}

Considering a MIMO system with: $n_{T}=1$ and $n_{R}$ varying from 2 to 22 , operating over Rayleigh fading channel, yielded the following results. More specifically, the MIMO channel's capacity over the Rayleigh fading channel was simulated using MATLAB software for different antenna populations and different values of SNR. The SNR values increased from $0 \mathrm{db}$ to $+35 \mathrm{db}$ at increments of $+5 \mathrm{db}$. Figure 4 depicts the channel's capacity using the receive diversity systems, as a function of the number of antennas $n_{R}$.

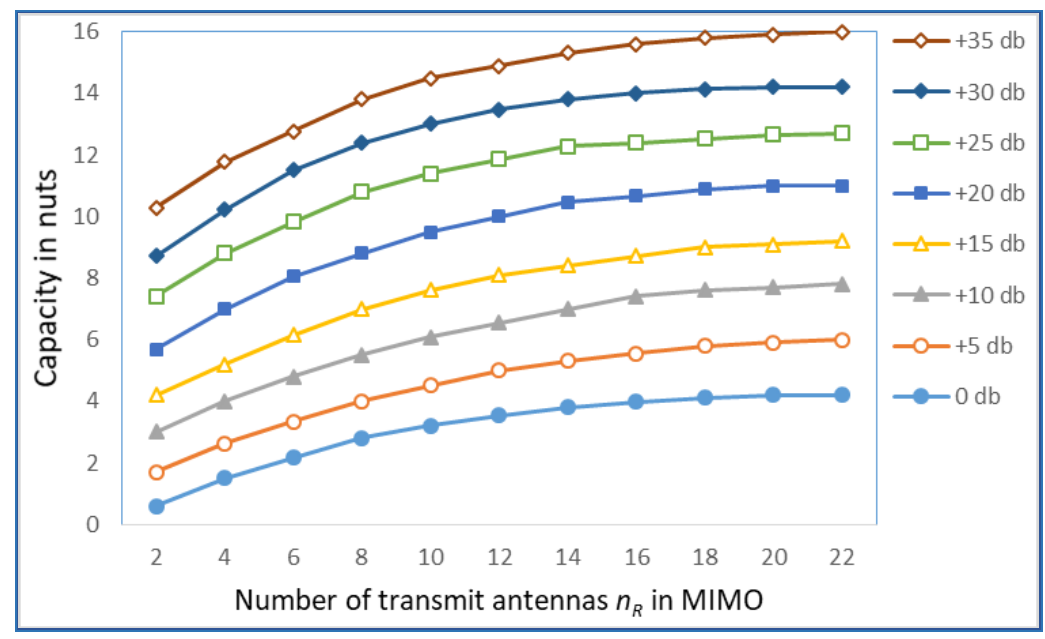

Figure 4. The channel capacity in nuts of MIMO systems versus the number of transmitting antennas, for various SNR levels.

Figure 4 shows that the higher the levels of the SNR value, the higher the magnitude Capacity in nuts of MIMO systems. However, when $n_{R} \geq 20$, the values of capacity for each SNR value level remain constant (saturated), although the number of antennas may increase

\subsection{Capacity Cost of MIMO systems (three different structures)}

Three MIMO system structures (scenarios) are selected as study examples here. The structures are the following:

Scenario (a): uses an exclusive one base-station, with $C_{B C}=1000$ Euros, $C_{E P C}=110$ Euros.

Scenario (b): uses an exclusive one base-station, with $C_{B C}=1000$ Euros, $C_{E P C}=150$ Euros.

Scenario (c): uses two base-station, with $C_{B C}=1000$ Euros, $C_{E P C}=110$ Euros.

In all of the above mentioned cases, the number of antennas is: $n_{T}=1$, while $n_{R}$ varies from 2 to 22, and the cost of each MIMO's antenna is considered equal to A (Euros). According to Section 3 , the corresponding Cost calculation was carried out for the three structural MIMO scenarios.

In Figure 5, the cost (Values x A (Euros)) of three different MIMO systems versus the number of antennas that are used on the receiving side is depicted. The number of antennas ranged from 2 to 22. Furthermore, A shows the cost per antenna in euros. 


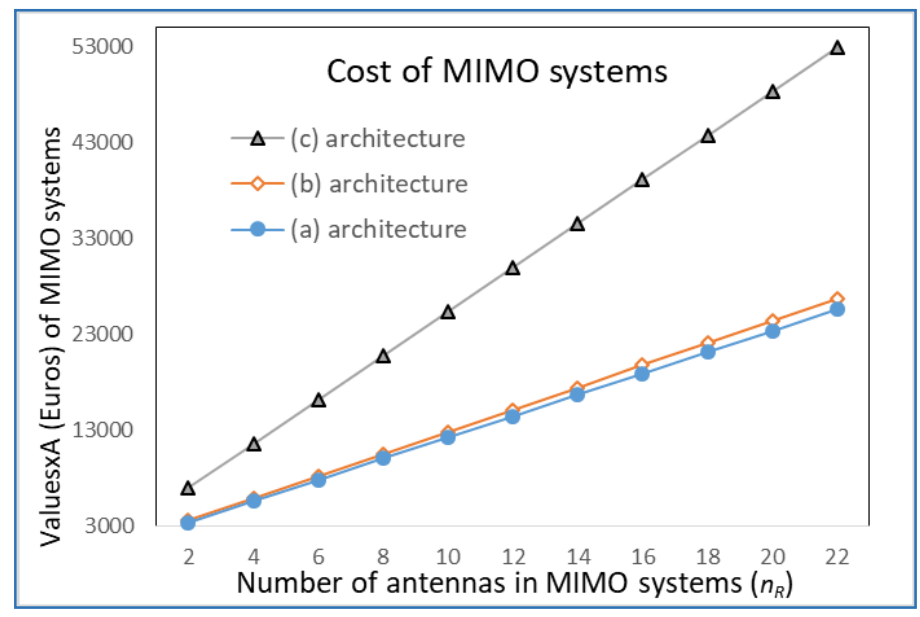

Figure 5. Fixed cost of MIMO systems versus antenna populations at the receiving side.

From Figure 5, it can be deduced that when the number of antennas is increased, the cost of each MIMO system increases linearly. However, the increment of cost becomes dramatic when an additional Base Station (BS) is deployed (scenario c), due to the corresponding complexity. Nevertheless, because of the linear increase in the cost factor, the corresponding normalized cost values of all three scenarios $(\mathrm{a}, \mathrm{b}$ and $\mathrm{c}$ ) are identical.

\subsection{Evaluating MIMO systems in terms of capacity and cost on a Rayleigh fading channel}

For the sake of this study, the above methodology is applied to various MIMO scenarios with different weights. The GEF indicator regarding the cost and the capacity factors of some MIMO systems is calculated. The optimal selection of the investigated systems is when they have a small cost and a high capacity. In the first one, the evaluation weights of the cost and capacity factors are equivalent. In the second scenario, the evaluation weights are considered as, and the third scenario has as weights the pair.

\subsubsection{Capacity and cost weighting equivalents}

Figure 6 plots the GEF versus the number of antennas that MIMO systems use for various levels of SNR, assuming that the MIMO system operates on a Rayleigh fading channel. In Figure 6, it is obvious that when the antenna population is greater than 10, the GEF factor increases so the solution deteriorates gradually (in terms of capacity and cost). Nevertheless, in the antenna population, the GEF factor is independent of the SNR levels. 
International Journal of Computer Networks \& Communications (IJCNC) Vol.12, No.1, January 2020

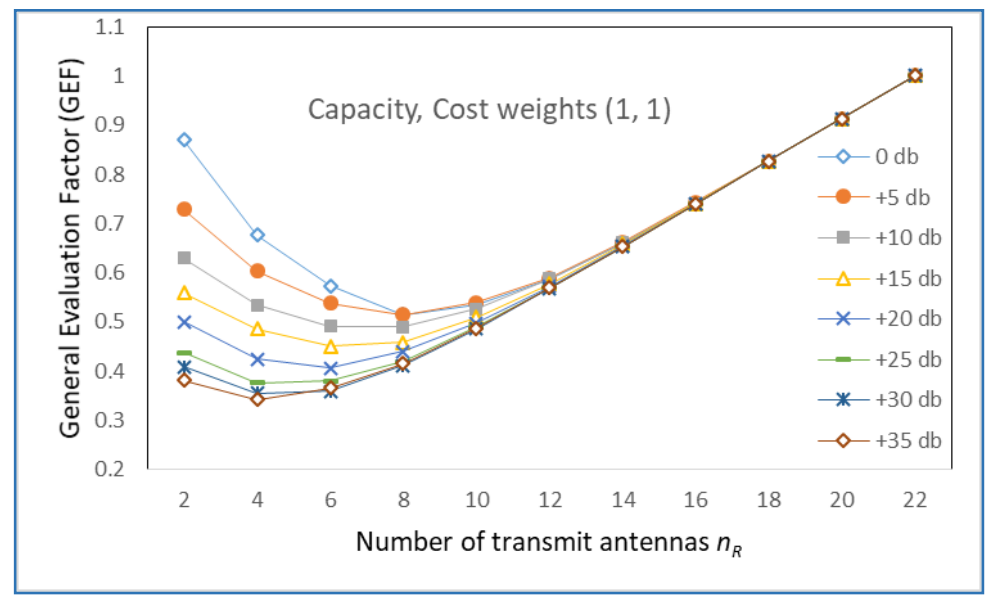

Figure 6. GEF for MIMO systems in terms of capacity and cost weighing equivalents

On the other hand, differentiation is observed when the antenna population is less than 10; particularly so when $n_{R} \leq 10$ and SNR level is greater than $20 \mathrm{~dB}$. Moreover, the optimum solution can be identified for antenna numbers as between 4 and 6 . In addition, when $n_{R} \leq 10$ and SNR level is less than $10 \mathrm{~dB}$, the most advantageous solution (considering the balance between capacity and cost factors) is almost 8 antennas ( 2 additional antennas are needed).

\subsubsection{Capacity and cost weight $(2,1)$}

In this second scenario, the capacity factor is considered with double gravity. Otherwise, the cost factor is degraded in relation to the system's capacity

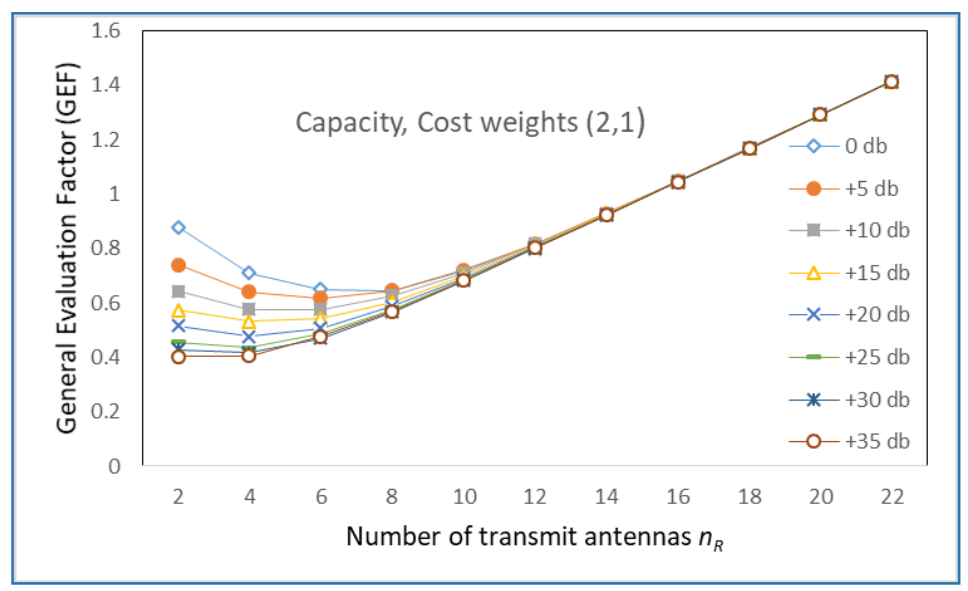

Figure 7. GEF for MIMO systems in terms of capacity and cost with their corresponding weights $(2,1)$

Figure 7 illustrates that when the antenna population becomes greater than 10, the GEF factor increases so the solution deteriorates gradually; despite this, the factor remains independent of SNR levels. On the other hand, when $n_{R} \leq 10$ and the SNR level is greater than $10 \mathrm{~dB}$, the best GEF values are approximately 0.6 and the corresponding number of $n_{R}$ is equal to 6. For SNR levels greater than $10 \mathrm{~dB}$, however, the optimum number of antennas on the receiving side is approximately 4 . 


\subsubsection{Capacity and cost weights $(1,2)$}

In the third hypothesis, the factor of the MIMO system, the cost is taken into account with double gravity (i.e. $w_{j}=2$ ), while the capacity factor is degraded (i.e. $w_{i}=1$ ).

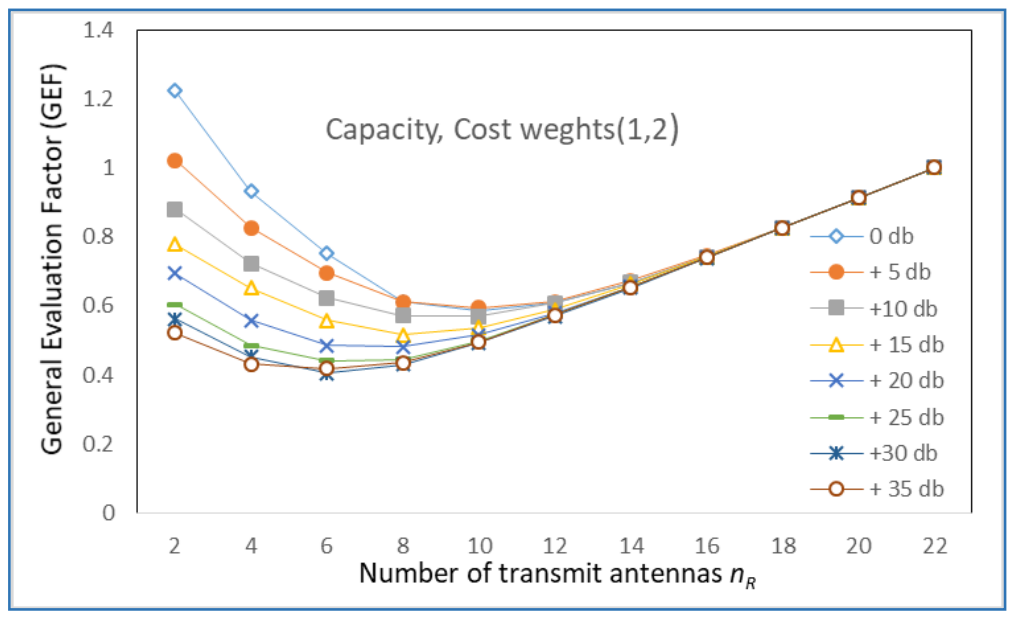

Figure 8. Evaluating MIMO systems in terms of capacity and cost with their corresponding weights $(1,2)$

Figure 8 indicates that when the antenna population on the receiving side becomes greater than 14, the GEF factor increases, and this causes the deterioration of the system (cost and performance factors). Nevertheless, when the population of antennas is large (>14), the GEF values are not affected by the SNR levels. The optimum solution in this scenario is presented when the SNR value is greater than $20 \mathrm{db}$. In this case, the best GEF value is approximately 0.4 , and the best possible number of antennas on the receiving side is 6 .

All the above findings involve MIMO systems that operate over the Rayleigh fading channel. Moreover, this analysis can be used in many other special cases of MIMO systems applications, such as embedded digital MIMO, digital radar [26] and so on.

\section{CONCLUSIONS AND FUture Work}

Nowadays, the demand for 5G technology has created a totally new variety of telecommunications, of which MIMO technology will certainly be a core component. MIMO technology includes antennas, which facilitates better signal transmission, achieves better coverage, and increases network capacity, as well as being ideal for bandwidth reallocation. MIMO networks are in general multi-factorial systems; indeed, almost every modern technological solution related to $5 \mathrm{G}$ technology is a multi-factorial proposal.

In this paper, a method assisting in the evaluation of multiple-magnitude dependent MIMO systems is presented. The method is exemplified in MIMO networks that operate over a Rayleigh fading channel. This specific scenario of MIMO systems is examined from a techno-economic perspective in terms of capacity and cost factors. Nevertheless, the method is a general contrivance that can be applied in a variety of ways and incorporate many factors, each with a separate weight. This study presents an improved view; however, the sheer amount of scientific scenarios that can be explored and optimized by this method are extensive. 
Future research activity on MIMO networks should focus on novel ways of using new technologies and infrastructures. New ideas for optimizing factors like power consumption, costs for back-hauling, and bandwidth, among others, should be investigated and weighed before their use. Moreover, other types of variable costs (e.g. daily operation and maintenance costs) also play a critical role in the cost formation. New cost models should be developed for all the key 5Genabled technologies, combining costs with the corresponding technology requirements. Furthermore, the research could focus on optimizing the number of MIMO transceivers and receivers on each side and weight and improve various contradictory parameters.

\section{REFERENCES}

[1] B. Vucetic, J. Yuan, Space-time coding. John Wiley \& Sons, 2003.

[2] B. Panzner, W. Zirwas, S. Dierks, M. Lauridsen, P.Mogensen, K. Pajukoski and D. Miao, "Deployment and implementation strategies for massive MIMO in 5G". in Proc. IEEE Globecom Workshops (GC Wkshps), Austin, TX, USA, Dec. 2014, pp. 346-351.

[3] C. Du, J. S. Thompson and Y. Petillot, "Predicted Detection Performance of MIMO Radar," in IEEE Signal Processing Letters, vol. 15, pp. 83-86, 2008.

[4] S. N. Sur, R. Bera and B. Maji, "Capacity Analysis of MIMO in Non-Uniform Nakagami Channel with Linear Receiver". IETE Journal of Research, vol. 63, pp.795-804, May 2017.

[5] T. J. Huang, "Analytical Investigation of Channel Capacity of UWB-MIMO Systems," International Journal of Communications, Network and System Sciences, vol.11 no.1, pp.1-8, Jan. 2018.

[6] A. F. Molisch, M. Steinbauer, M. Toeltsch, E. Bonek and R. S. Thoma, "Capacity of MIMO systems based on measured wireless channels," in IEEE Journal on Selected Areas in Communications, vol. 20, no. 3, pp. 561-569, April 2002.

[7] W. Q. Malik and D. J. Edwards, "Measured MIMO Capacity and Diversity Gain With Spatial and Polar Arrays in Ultrawideband Channels," in IEEE Transactions on Communications, vol. 55, no. 12, pp. 2361-2370, Dec. 2007.

[8] K. Tiwari, D. S.Saini, and S. V. Bhooshan, "On the capacity of MIMO Weibull-Gamma fading channels in low SNR regime," Journal of Electrical and Computer Engineering, 2016.

[9] K. Tiwari, D. S. Saini, and S. V. Bhooshan, "Antenna selection for MIMO systems over WeibullGamma fading channel". Perspectives in Science, vol. 8, pp. 475-478, Sept. 2016.

[10] L. Malviya, R. K. Panigrahi and M. V. Kartikeyan, "Four Element Planar MIMO Antenna Design for Long-Term Evolution Operation,” Journal IETE Journal of Research, vol. 64, no. 3, pp. 367-373, Aug 2017

[11] M. Abdul Haleem, "On the Capacity and Transmission Techniques of Massive MIMO Systems," Wireless Communications and Mobile Computing, pp. 1-9, July 2018.

[12] L. Lu, G. Y. Li, A. L. Swindlehurst, A. Ashikhmin and R. Zhang, "An Overview of Massive MIMO: Benefits and Challenges," in IEEE Journal of Selected Topics in Signal Processing, vol. 8, no. 5, pp. 742-758, Oct. 2014

[13] G. Lee and Y. Sung, "A New Approach to User Scheduling in Massive Multi-User MIMO Broadcast Channels", in IEEE Transactions on Communications, vol. 66, no. 4, pp. 1481-1495, April 2018.

[14] P. Harris, M. Beach, S. Armour, and I. Mings, "From MIMO to Massive MIMO", MICROWAVE JOURNAL, vol. 60, no. 9, pp.22-42, Sept. 2017.

[15] E. Björnson, J. Hoydis and L. Sanguinetti, "Massive MIMO Has Unlimited Capacity", in IEEE Transactions on Wireless Communications, vol. 17, no. 1, pp. 574-590, Jan. 2018.

[16] J. Xue, T. Ratnarajah, C. Zhong and C. Wen, "Reliability Analysis for Large MIMO Systems", in IEEE Wireless Communications Letters, vol. 3, no. 6, pp. 553-556, Dec. 2014.

[17] A. Kumar, and P. Vardhan, "Design, Simulation \& Concept Verification of $4 \times 4,8 \times 8$ MIMO with ZF, MMSE and BF Detection Schemes," Electrical, Control and Communication Engineering, vol. 13, no. 1, pp. 69-74, 2017.

[18] W. Tan, G. Xu, E. De Carvalho, M. Zhou, L. Fan, and C. Li, "Low cost and high efficiency hybrid architecture massive MIMO systems based on DFT processing," Wireless Communications and Mobile Computing, pp. 2018.

[19] G. Caire, S. A. Ramprashad and H. C. Papadopoulos, "Rethinking network MIMO: Cost of CSIT, performance analysis, and architecture comparisons," 2010 Information Theory and Applications Workshop (ITA), San Diego, CA, , pp. 1-10, Jan 2010. 
[20] L. Liu, C. Oestges, J. Poutanen, K. Haneda, P.Vainikainen, F. Quitin, and P. De Doncker, "The COST 2100 MIMO channel model," in IEEE Wireless Communications, vol. 19, no. 6, pp. 92-99, December 2012.

[21] C. Bouras, S.Kokkalis, A. Kollia, and A. Papazois, "Techno-economic comparison of MIMO and DAS cost models in 5G networks", Wireless Networks, pp. 1-15, 2018.

[22] G. J. Foschini, and M. J. Gans, "On limits of wireless communications in a fading environment when using multiple antennas”, Wireless personal communications, vol. 6, no. 3, pp. 311-335, 1998.

[23] E. Telatar, "Capacity of multi-antenna Gaussian channels", European transactions on telecommunications, vol. 10, no. 6, pp. 585-95, Nov 1999.

[24] J. D. Garofalakis, and E. Stergiou, "An Approximate Analytical Performance Model for Multistage Interconnection Networks with Backpressure Blocking Mechanism”, JCM, vol. 5, no. 3, pp. 247-261, 2010.

[25] D. Vasiliadis, G. Rizos, and Costas Vassilakis, "Performance study of multilayered multistage interconnection networks under hotspot traffic conditions", Journal of Computer Systems, Networks, and Communications, pp. 1-11, January 2010.

[26] S. Shome, R. Bera, B. Maji, S. N. Sur, and S. Bera, "Embedded Digital MIMO Radar Using SDR for Target Detection and RCS Measurement," Journal IETE Journal of Research, vol. 62, no. 1, pp. 100105, Sep 2015.

[27] T. S. Rappaport, Wireless communications: principles and practice, New Jersey: prentice hall PTR, 1996.

\section{Authors}

Eleftherios Stergiou is associate professor in the Department of Informatics and Telecommunications at the University of Ioannina (former Department of Informatics and Telecommunications University at the Technological Educational Institute of Epirus). He has been joined in this department since 2000. He received the B.S. degree in electrical engineering from NTUA, Athens Greece, and he finished his postgraduate studies at the Computer Science Department of the University of Sheffield (1998) and

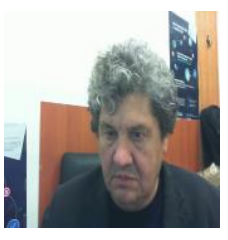
his Ph.D. at the University of Patras. His research interests on performance evaluation of networks were pursued by publishing papers in international journals. Among these interests, computing analytical methods, interconnection networks, parallel and distributed systems, and high-speed networks are included. Mr E. Stergiou is a member of the IEEE Computer Society.

Constantinos Angelis is Professor of Microelectronic Devices and Telecommunication Systems at the Department of Informatics and Telecommunications, University of Ioannina. His research interests are in Analog and mixed signal devices, circuits and systems design, Micro-electronic devices, Electrical \& Noise Characterization, Antenna Systems Design for Cellular \& Satellite applications, Wireless communication systems, designs and implementations, and MIMO systems. He has published over 40 papers in international journals and conferences and chapter books. He is member of IEEE and

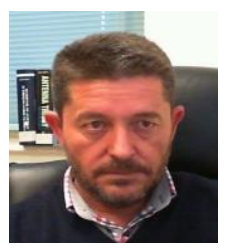
EuMA.

Spiridoula V. Margariti received her Ph.D. in 2017 from the Department of Computer Science and Engineering, University of Ioannina, Greece. She received her MSc and BSc in Computer Science in 2002 and 2007 respectively, from the Department of Computer Science and Engineering, University of Ioannina, Greece. Her research interests include communication and wireless networks, complex and distributed systems, p2p data management and overlay protocols. 